\title{
PROBLEMS IN MODELLING CHARGE OUTPUT ACCELEROMETERS
}

\section{Krzysztof Tomezyk}

Cracow University of Technology, Faculty of Electrical and Computer Engineering, Warszawska 24, 31 -155 Kraków, Poland \tomczyk@pk.edu.pl, +48 12628 2543)

\begin{abstract}
The paper presents major issues associated with the problem of modelling change output accelerometers. The presented solutions are based on the weighted least squares (WLS) method using transformation of the complex frequency response of the sensors. The main assumptions of the WLS method and a mathematical model of charge output accelerometers are presented in first two sections of this paper. In the next sections applying the WLS method to estimation of the accelerometer model parameters is discussed and the associated uncertainties are determined. Finally, the results of modelling a PCB357B73 charge output accelerometer are analysed in the last section of this paper. All calculations were executed using the MathCad software program. The main stages of these calculations are presented in Appendices A-E.
\end{abstract}

Keywords: weighted least square method, charge output accelerometer, mathematical modelling, parameter estmation.

(C) 2016 Polish Academy of Sciences. All rights reserved

\section{Introduction}

Modelling accelerometers with charge output, based on the measured data of frequency characteristics, should be carried out by taking into account a charge-to-voltage conversion system. Therefore, it is necessary to combine the accelerometer transfer function and the transfer function related to both the voltage amplifier and the connecting cable [1]. Such an approach can guarantee proper estimation of the model parameters.

In many cases, the accelerometers with charge output are modelled in the same way as the accelerometers with voltage output, which is a significant simplification of the estimation procedure. This modelling is usually carried out by means of the WLS method which enables simultaneous approximation of the amplitude and phase characteristics. The majority of studies discuss the nonlinear version of this method [2-11]. It is implemented in a recursive way and is aimed at minimizing the objective function by using one of gradient type methods (mostly employing the Levenberg-Marquardt algorithm). However, the problems related to proper determination of the regularization parameter as well as the significant computational difficulties resulting from the necessity to determine Jakobian matrix have arisen.

In spite of the above limitations, the linear version of WLS method provides satisfactory results in the widely understood sense of modelling measurement systems. This procedure is based on the Monte Carlo (MC) method which is used to determine the covariance matrix by performing a series of draws from the normal Gaussian distribution. This distribution is defined by the statistical metrics (mean and standard deviation) which are calculated from the measured data of both frequency characteristics [12]. The matrix-vector equation which meets the linear WLS method requirements is determined on the basis of two complex frequency responses. The first one results from the measurement data, while the second one refers 
to an assumed parametric model of the accelerometer [13-14]. A straightforward and computationally efficient linear WLS method based on the inverse complex frequency responses is discussed in detail in [15]. This method was applied for modelling Endevco 2270 and Brüel\&Kjær 8305 charge output accelerometers and - as a result - the values of parameters and associated uncertainties of the sensors were obtained. However, the complex frequency responses were calculated on the basis of a mathematical model related to the accelerometers with voltage output [16-18]. Both the voltage amplifier and the connecting cable were modeled as a low-pass filter and their influence on the modelling output was neglected.

To omit the above simplification, a total system model including a charge output accelerometer, an amplifier and a connecting cable is considered in this paper.

\section{Applied modelling method}

The method discussed in this section can be applied for modelling sensors whose complex frequency response is presented by:

$$
K(\omega, \boldsymbol{\theta})=\frac{a_{0}+a_{1} j \omega-a_{2} \omega^{2}+\ldots+a_{m}(j \omega)^{m}}{1+b_{1} j \omega-b_{2} \omega^{2}+\ldots+b_{r}(j \omega)^{r}}
$$

where:

$$
\boldsymbol{\theta}=\left[a_{0}, a_{1}, a_{2}, \ldots, a_{m}, b_{1}, b_{2}, \ldots, b r\right]
$$

is a vector of unknown model parameters, and $m<r$. In turn, taking into account the measument points of amplitude $A\left(\omega_{n}\right)$ and phase $\Phi\left(\omega_{n}\right)$ characteristics, the complex frequency response is:

$$
K\left(\omega_{n}\right)=A\left(\omega_{n}\right) \exp \left[j \Phi\left(\omega_{n}\right)\right]=R\left(\omega_{n}\right)+I\left(\omega_{n}\right), \quad n=0,1, \ldots, N-1,
$$

where $N$ denotes the number of measurement points, and $R\left(\omega_{n}\right)$ and $I\left(\omega_{n}\right)$ are the real and the imaginary parts of this frequency response, respectively.

Let us also introduce the numerator $N$ and the de-numerator $D$ of the relation (1) by means of their real and imaginary representation in accordance with the measured frequencies $\omega_{n}[14$ 15]. Then, we have:

$$
K\left(\omega_{n}, \boldsymbol{\theta}\right)=\frac{N_{R}\left(\omega_{n}, \boldsymbol{\theta}\right)+j N_{I}\left(\omega_{n}, \boldsymbol{\theta}\right)}{1+D_{R}\left(\omega_{n}, \boldsymbol{\theta}\right)+j D_{I}\left(\omega_{n}, \boldsymbol{\theta}\right)} .
$$

The best estimation of model parameters is obtained when the deviation between the measured (3) and estimated (4) frequency responses takes the minimum value. Then, we can assume the close equality of (3) and (4), as follows:

$$
\frac{N_{R}\left(\omega_{n}, \boldsymbol{\theta}\right)+j N_{I}\left(\omega_{n}, \boldsymbol{\theta}\right)}{1+D_{R}\left(\omega_{n}, \boldsymbol{\theta}\right)+j D_{I}\left(\omega_{n}, \boldsymbol{\theta}\right)} \cong R\left(\omega_{n}\right)+j I\left(\omega_{n}\right)=\varepsilon_{R}\left(\omega_{n}\right)+j \varepsilon_{I}\left(\omega_{n}\right) .
$$

Simple transformation of (5) yields the estimation errors:

$$
\varepsilon_{R}\left(\omega_{n}\right)=N_{R}\left(\omega_{n}, \boldsymbol{\theta}\right)-R\left(\omega_{n}\right) D_{R}\left(\omega_{n}, \boldsymbol{\theta}\right)+I\left(\omega_{n}\right) D_{I}\left(\omega_{n}, \boldsymbol{\theta}\right)
$$

and

$$
\varepsilon_{I}\left(\omega_{n}\right)=N_{I}\left(\omega_{n}, \boldsymbol{\theta}\right)-I\left(\omega_{n}\right) D_{R}\left(\omega_{n}, \boldsymbol{\theta}\right)-R\left(\omega_{n}\right) D_{I}\left(\omega_{n}, \boldsymbol{\theta}\right) .
$$

In an analogous way, based on (1), the following relations can be obtained:

$$
\begin{gathered}
N_{R}\left(\omega_{n}, \boldsymbol{\theta}\right)=a_{0}-a_{2} \omega_{n}^{2}+a_{4} \omega_{n}^{4}-\ldots \\
N_{I}\left(\omega_{n}, \boldsymbol{\theta}\right)=a_{1} \omega_{n}-a_{3} \omega_{n}^{3}+a_{5} \omega_{n}^{5}-\ldots
\end{gathered}
$$




$$
\begin{aligned}
& D_{R}\left(\omega_{n}, \boldsymbol{\theta}\right)=-b_{2} \omega_{n}^{2}+b_{4} \omega_{n}^{4}-b_{6} \omega_{n}^{6}+\ldots \\
& D_{I}\left(\omega_{n}, \boldsymbol{\theta}\right)=b_{1} \omega_{n}-b_{3} \omega_{n}^{3}+b_{5} \omega_{n}^{5}-\ldots
\end{aligned}
$$

Substitution (8) into (6) and (7) yields:

$$
\begin{aligned}
\varepsilon_{R}\left(\omega_{n}\right) & =a_{0}-a_{2} \omega_{n}^{2}+a_{4} \omega_{n}^{4}-\ldots+R\left(\omega_{n}\right) b_{2} \omega_{n}^{2}-R\left(\omega_{n}\right) b_{4} \omega_{n}^{4}+R\left(\omega_{n}\right) b_{6} \omega_{n}^{6}-\ldots+ \\
& +I\left(\omega_{n}\right) b_{1} \omega_{n}-I\left(\omega_{n}\right) b_{3} \omega_{n}^{3}+I\left(\omega_{n}\right) b_{5} \omega_{n}^{5}-\ldots
\end{aligned}
$$

and

$$
\begin{gathered}
\varepsilon_{I}\left(\omega_{n}\right)=a_{1} \omega_{n}-a_{3} \omega_{n}^{3}+a_{5} \omega_{n}^{5}-\ldots+I\left(\omega_{n}\right) b_{2} \omega_{n}^{2}+ \\
-I\left(\omega_{n}\right) b_{4} \omega_{n}^{4}+I\left(\omega_{n}\right) b_{6} \omega_{n}^{6}-\ldots-R\left(\omega_{n}\right) b_{1} \omega_{n}+ \\
+R\left(\omega_{n}\right) b_{3} \omega_{n}^{3}-R\left(\omega_{n}\right) b_{5} \omega_{n}^{5}+\ldots
\end{gathered}
$$

The estimation $\widetilde{\boldsymbol{\theta}}$ of the vector (2) is calculated using the formula:

$$
\widetilde{\boldsymbol{\theta}}=\left(\boldsymbol{\Psi}^{T} \boldsymbol{\Sigma}^{-1} \boldsymbol{\Psi}\right)^{-1} \boldsymbol{\Psi}^{T} \boldsymbol{\Sigma}^{-1} \mathbf{Y},
$$

which represents the linear WLS method. The $2 N$-dimensional vector $\mathbf{Y}$ is constructed based on (3) and it takes the following form:

$$
\mathbf{Y}^{T}=\left[R\left(\omega_{0}\right) I\left(\omega_{0}\right) R\left(\omega_{1}\right) I\left(\omega_{1}\right) \ldots R\left(\omega_{N}\right) I\left(\omega_{N}\right)\right],
$$

while the $2 N x(m+1+r)$-dimensional matrix $\boldsymbol{\Psi}$ is represented by (see Appendix A):

$$
\boldsymbol{\Psi}^{T}=\left[\begin{array}{llll}
\boldsymbol{\psi}_{0}^{T} & \boldsymbol{\psi}_{1}^{T} & \ldots & \boldsymbol{\psi}_{N}^{T}
\end{array}\right] .
$$

Successive rows of this matrix are developed by splitting up (9) - (10) according to the order of parameters in the vector (2), as follows:

$$
\boldsymbol{\psi}_{n}=\left[\begin{array}{cc}
1 & 0 \\
0 & \omega_{n} \\
-\omega_{n}^{2} & 0 \\
0 & -\omega_{n}^{3} \\
\omega_{n}^{4} & 0 \\
\vdots & \vdots \\
I\left(\omega_{n}\right) \omega_{n} & -R\left(\omega_{n}\right) \omega_{n} \\
R\left(\omega_{n}\right) \omega_{n}^{2} & I\left(\omega_{n}\right) \omega_{n}^{2} \\
-I\left(\omega_{n}\right) \omega_{n}^{3} & R\left(\omega_{n}\right) \omega_{n}^{3} \\
-R\left(\omega_{n}\right) \omega_{n}^{4} & -I\left(\omega_{n}\right) \omega_{n}^{4} \\
\vdots & \vdots
\end{array}\right] .
$$

The diagonal covariance matrix $\boldsymbol{\Sigma}$, whose dimension is equal to $2 N x 2 N$ is: 


$$
\boldsymbol{\Sigma}=\left[\begin{array}{cccc}
\Sigma_{0} & 0 & \ldots & 0 \\
0 & \Sigma_{1} & \ldots & 0 \\
\vdots & \vdots & \ddots & \vdots \\
0 & 0 & \ldots & \Sigma_{2 N}
\end{array}\right]
$$

According to the standard [12], it is recommended to apply the MC method to determine this matrix in two stages [15].

During the first stage the matrices:

$$
\begin{gathered}
v_{A_{n, m}}=A\left(\omega_{n}\right)+u\left(A_{\mathrm{n}}\right) \varepsilon_{A_{n, m}}, \\
v_{\Phi_{n, m}}=\Phi\left(\omega_{n}\right)+u\left(\Phi_{n}\right) \varepsilon_{\Phi_{n, m}}, \quad m=0,1, \ldots, M-1
\end{gathered}
$$

are calculated, where $M$ denotes the number of MC trials. The vectors:

$$
u\left(A_{\mathrm{n}}\right)=\sqrt{\frac{1}{N(N-1)} \sum_{n=0}^{N-1}\left(A\left(\omega_{n}\right)-\frac{1}{N} \sum_{n=0}^{N-1} A\left(\omega_{n}\right)\right)^{2}}
$$

and

$$
u\left(\Phi_{n}\right)=\sqrt{\frac{1}{N(N-1)} \sum_{n=0}^{N-1}\left(\Phi\left(\omega_{n}\right)-\frac{1}{N} \sum_{n=0}^{N-1} \Phi\left(\omega_{n}\right)\right)^{2}},
$$

contain the uncertainties associated with the measured vectors $A\left(\omega_{n}\right)$ and $\Phi\left(\omega_{n}\right)$, while the vectors $\varepsilon_{A}$ and $\varepsilon_{\Phi}$ include the random variables drawn from the Gaussian normal distribution (e.g. the Box-Muller basic form - see Appendix B) with means and standard deviations calculated on the basis of vectors $A\left(\omega_{n}\right)$ and $\Phi\left(\omega_{n}\right)$ [19].

The second stage refers to determination of the matrix:

$$
\begin{aligned}
& \mathbf{V}_{(2 N, M)}= \\
& =\left[\begin{array}{ccc}
\operatorname{Re}\left[v_{A_{0,0}} \exp \left(j v_{\Phi(0,0)}\right)\right] & \cdots & \operatorname{Re}\left[v_{A}, M-1\right. \\
\vdots & \cdots & \exp \left(j v_{\Phi}, M-1\right. \\
\operatorname{Re}\left[v_{A_{N-1,0}} \exp \left(j v_{\Phi(N-1,0)}\right)\right] & \cdots & \operatorname{Re}\left[v_{A}{ }_{N-1, M-1} \exp \left(j v_{\Phi}{ }_{N-1, M-1}\right)\right] \\
\operatorname{Im}\left[v_{A, 0} \exp \left(j v_{\Phi(0,0)}\right)\right] & \cdots & \operatorname{Im}\left[v_{A}, M-1\right. \\
\vdots & \cdots & \exp \left(j v_{\Phi} 0, M-1\right. \\
\left.\exp \left(j v_{\Phi(N-1,0)}\right)\right] & \cdots & \operatorname{Im}\left[v_{A_{N-1, M-1}} \exp \left(j v_{\Phi}{ }_{N-1, M-1}\right)\right]
\end{array}\right] .
\end{aligned}
$$

Particular elements $\Sigma_{n}$ of the diagonal in matrix $\boldsymbol{\Sigma}$ are determined as the inverse of the variance for each row of the matrix $\mathbf{V}^{T}$ (see Appendix C).

\section{Mathematical model of charge output accelerometer}

The mathematical model of charge output accelerometer is represented by its complex frequency responses which can be directly derived from the differential equation:

$$
m \ddot{y}(t)+r \dot{z}(t)+k z(t)=0 .
$$


This equation is obtained on the basis of corresponding mechanical construction of a seismic mass accelerometer shown in Fig. 1, where the following notations are used: $z(t)$ - the relative mass displacement (relative output), $y(t)$ - the absolute mass displacement (absolute output), $x(t)$ - the vibration (excitation), $m[\mathrm{~kg}]$ - the seismic mass, $r[\mathrm{~kg} \cdot \mathrm{m} / \mathrm{s}]$ - a dumping coefficient, $k[N / m]$ - a spring constant, $m \ddot{y}(t)$ - the inertial force, $r \dot{z}(t)$ - the dumping force, $k z(t)$ - the elastic force.

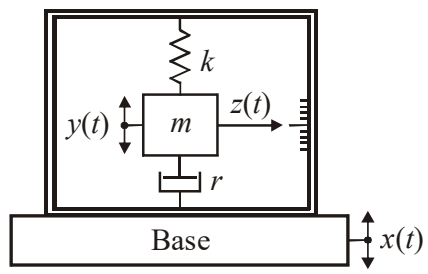

Fig. 1. The mechanical construction of a seismic mass accelerometer.

Analysing Fig. 1, we have:

$$
y(t)=z(t)+x(t),
$$

Substitution (21) into (20) gives:

$$
m \ddot{z}(t)+r \dot{z}(t)+k z(t)=-m \dot{x}(t) .
$$

The response of charge output accelerometer to the force $F(t)$ which acts onto the quartz crystal represents the absolute mass displacement. Unlike to the seismic mass accelerometer, it implies the following substitution in (20):

After this substitution, we have:

$$
z(t)=y(t)-x(t) .
$$

$$
m \ddot{y}(t)+r \dot{y}(t)+k y(t)=r \dot{x}(t)+k x(t) .
$$

Transformation of (24) into the $s$-domain yields:

and finally

$$
\frac{m}{k} s^{2} Y(s)+\frac{r}{k} s Y(s)+Y(s)=\frac{r}{k} s X(s)+X(s)
$$

where:

$$
\frac{1}{\omega_{0}^{2}} s^{2} Y(s)+\frac{2 \beta}{\omega_{0}} s Y(s)+Y(s)=X(s)\left(\frac{2 \beta}{\omega_{0}} s+1\right),
$$

$$
\omega_{0}=\sqrt{\frac{k}{m}}, \beta=\frac{r}{2 \sqrt{k m}} .
$$

The force $F(t)$ generates the electric charge $Q(t)$ :

$$
Q(t)=k_{p} F(t),
$$

where $k_{p}=2.2 \cdot 10^{-12}[C / N]$ is a piezoelectric constant.

The mechanical construction of a charge output accelerometer is shown in Fig. 2. 


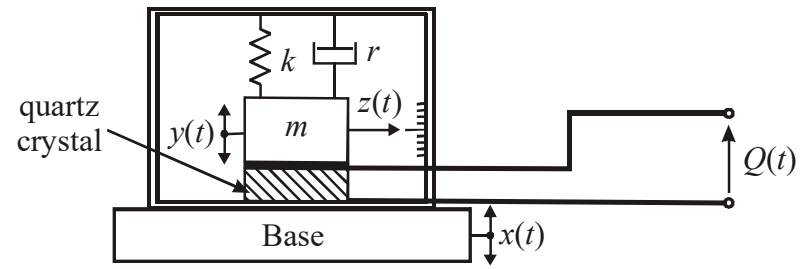

Fig. 2. The mechanical construction of a charge output accelerometer.

The force is proportional to the absolute acceleration according to:

$$
F(t)=m \ddot{y}(t) .
$$

Substituting (29) into (28) and then transforming it into the $s$-domain, we have:

$$
Y(s)=\frac{Q(s)}{s^{2} m k_{p}} .
$$

Taking into account (30) and (26), we obtain the following complex frequency response:

$$
K_{Q}(\omega, \boldsymbol{\theta})=S_{Q} \frac{\omega_{0}^{2}+2 j \beta \omega_{0} \omega}{\omega_{0}^{2}-\omega^{2}+2 j \beta \omega_{0} \omega},
$$

where: $S_{Q}=k_{p} S_{m}\left[C / m \cdot s^{2}\right]$ is the charge sensitivity; $\beta$ and $\omega_{0}$ represent the damping ratio and the non-damped natural frequency, respectively, while $S_{m}=m[\mathrm{~kg}]$ is the mechanical sensitivity.

Figure 3 shows an equivalent circuit model of a charge output accelerometer. This circuit includes a voltage amplifier and a low-noise coaxial cable.

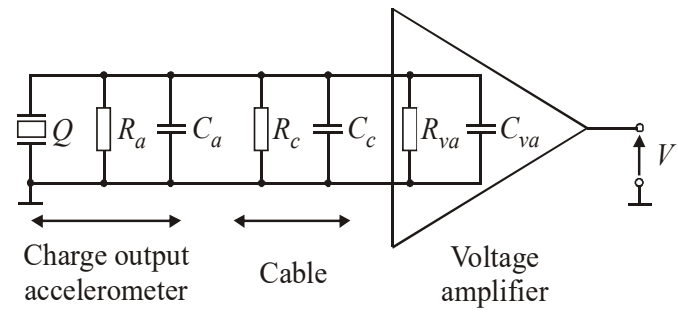

Fig. 3. An equivalent circuit model of a charge output accelerometer.

A simplified circuit model of a charge output accelerometer is shown in Fig. 4. Based on this model we can calculate the total resistance and capacitance in a simple way [17]:

and

$$
1 / R_{t}=1 / R_{a}+1 / R_{c}+1 / R_{v a}
$$

$$
C_{t}=C_{a}+C_{c}+C_{v a}
$$

In (32) - (33) there are: the accelerometer internal resistance and capacitance $R_{a}$ and $C_{a}$, the resistance between the cable screen and the centre conductor $R_{c}$, the cable capacitance $C_{c}$, the resistance $R_{v a}$ and capacitance $C_{v a}$. of the voltage amplifier input. 


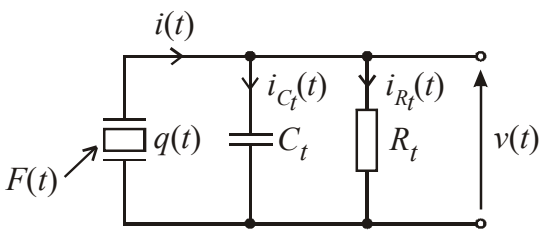

Fig. 4. A simplified circuit model of a charge output accelerometer.

Analysing the circuit model shown in Fig. 4, it is easy to obtain:

$$
\frac{V(s)}{Q(s)}=\frac{s R_{t}}{s R_{t} C_{t}+1} .
$$

The corresponding complex frequency response has the form:

$$
K(\omega, \boldsymbol{\theta})=\frac{-2 \frac{S_{V} \beta \tau}{\omega_{0}} \omega^{2}+j S_{V} \tau \omega}{1-\left(\frac{1}{\omega_{0}^{2}}+2 \frac{\beta \tau}{\omega_{0}}\right) \omega^{2}+j\left[\left(2 \frac{\beta}{\omega_{0}}+\tau\right) \omega-\frac{\tau}{\omega_{0}^{2}} \omega^{3}\right]},
$$

where $S_{V}=S_{m} S_{e}\left[\mathrm{~V} / \mathrm{m} \cdot \mathrm{s}^{2}\right]$ is the voltage sensitivity determined by the product of the mechanical and electrical sensitivities, while $\tau=R_{t} C_{t}[s]$ is a time constant. The electrical sensitivity $S_{e}=k_{p} / C_{t}[V / N]$ and the time constant result from the electrical properties of the charge-to-voltage converter.

Let us present (35) in a form related to the model (1). Then, we have:

where:

$$
K(\omega, \boldsymbol{\theta})=\frac{j a_{1} \omega-a_{2} \omega^{2}}{1+j b_{1} \omega-b_{2} \omega^{2}-j b_{3} \omega^{3}},
$$

$$
a_{1}=S_{V} \tau, a_{2}=2 \frac{S_{V} \beta \tau}{\omega_{0}}, b_{1}=2 \frac{\beta}{\omega_{0}}+\tau, b_{2}=\left(\frac{1}{\omega_{0}^{2}}+2 \frac{\beta \tau}{\omega_{0}}\right), b_{3}=\frac{\tau}{\omega_{0}^{2}}
$$

are functional relations between the accelerometer parameters.

\section{Modelling charge output accelerometer}

In this section, the modelling method presented in Section 2 were used to model the charge output accelerometers. The complex frequency response (36) does not contain a constant $a_{0}$ in the numerator. In order to apply the linear WLS, it is necessary to extend the numerator with this constant. Hence, we have:

and

$$
K(\omega, \boldsymbol{\theta})=\frac{a_{0}+j a_{1} \omega-a_{2} \omega^{2}}{1+j b_{1} \omega-b_{2} \omega^{2}-j b_{3} \omega^{3}}
$$

$$
\boldsymbol{\theta}=\left[a_{0}, a_{1}, a_{2}, b_{1}, b_{2}, b_{3}\right] .
$$

Based on (38), the real and imaginary parts of numerator and de-numerator are as follows: 


$$
\begin{gathered}
N_{R}\left(\omega_{n}, \boldsymbol{\theta}\right)=a_{0}-a_{2} \omega_{n}^{2} \\
N_{I}\left(\omega_{n}, \boldsymbol{\theta}\right)=a_{1} \omega_{n} \\
D_{R}\left(\omega_{n}, \boldsymbol{\theta}\right)=-b_{2} \omega_{n}^{2} \\
D_{I}\left(\omega_{n}, \boldsymbol{\theta}\right)=b_{1} \omega_{n}-b_{3} \omega_{n}^{3},
\end{gathered}
$$

whereas both parts of errors are represented by:

$$
\begin{gathered}
\varepsilon_{R}\left(\omega_{n}\right)=a_{0}-a_{2} \omega_{n}^{2}+R\left(\omega_{n}\right) b_{2} \omega_{n}^{2}+I\left(\omega_{n}\right) b_{1} \omega_{n}-I\left(\omega_{n}\right) b_{3} \omega_{n}^{3}, \\
\varepsilon_{I}\left(\omega_{n}\right)=a_{1} \omega_{n}+I\left(\omega_{n}\right) b_{2} \omega_{n}^{2}-R\left(\omega_{n}\right) b_{1} \omega_{n}+R\left(\omega_{n}\right) b_{3} \omega_{n}^{3}
\end{gathered}
$$

and the corresponding submatrices are as follows:

$$
\boldsymbol{\psi}_{n}=\left[\begin{array}{cc}
1 & 0 \\
0 & \omega_{n} \\
-\omega_{n}^{2} & 0 \\
I\left(\omega_{n}\right) \omega_{n} & -R\left(\omega_{n}\right) \omega_{n} \\
R\left(\omega_{n}\right) \omega_{n}^{2} & I\left(\omega_{n}\right) \omega_{n}^{2} \\
-I\left(\omega_{n}\right) \omega_{n}^{3} & R\left(\omega_{n}\right) \omega_{n}^{3}
\end{array}\right] .
$$

It results from (42) that the constant $a_{0}$ appears in the vector $\widetilde{\boldsymbol{\theta}}$. It is a disadvantage of the above modelling method because any recalculation of the parameters of model (35) by traforming (37) is not possible. However, due to a high accuracy of the parameter estimation, the presented procedure can be very useful for the modelling used to determine maximum dynamic errors [16-18] as well as for the deconvolution algorithms [20-21]. In both cases, the accuracy of modelling is usually better than that of the construction of accelerometer model.

\section{Calculation of parameter uncertainties}

The uncertainties associated with the parameters of model (38) are calculated as follows [15] (see Appendix D):

- determine the vector:

$$
\hat{\boldsymbol{\theta}}^{m}=\widetilde{\boldsymbol{\theta}}+\Delta \boldsymbol{\varepsilon}^{m},
$$

where $\Delta \boldsymbol{\varepsilon}^{m}$ is a random vector drawn from the multidimensional normal distribution with mean equal to zero and the covariance matrix defined by:

$$
\boldsymbol{\Sigma}_{u}=\left(\boldsymbol{\Psi}^{T} \boldsymbol{\Sigma}^{-1} \boldsymbol{\Psi}\right)^{-1} ;
$$

- calculate the covariance matrix $\boldsymbol{\Sigma}_{\hat{\boldsymbol{\theta}}}$ associated with uncertainties $\hat{\boldsymbol{\theta}}$ on the basis of:

$$
\boldsymbol{\Sigma}_{\hat{\boldsymbol{\theta}}}=\left[\frac{1}{N-1} \sum_{n=0}^{N-1}\left\{\left(\hat{\theta}^{T}\right)_{n, i}-\frac{1}{N} \sum_{n=0}^{N-1}\left(\hat{\theta}^{T}\right)_{n, i}\right\}^{2}\right]^{T}, \quad i=0,1,2 ;
$$

- calculate the uncertainties $\hat{\boldsymbol{\theta}}$ associated with parameters (39) as the root squares of diagonal elements of matrix $\boldsymbol{\Sigma}_{\hat{\boldsymbol{\theta}}}$. 


\section{Results of accelerometer modelling}

The procedures discussed in Sections 2-5 were applied to modelling a PCB357B73 type charge output accelerometer. The main parameters of this sensor can be found in the corresponding datasheet. Hence, we have:

- charge sensitivity $S_{Q}=10.2\left[\mathrm{pC} / \mathrm{m} \cdot \mathrm{s}^{2}\right] \pm 5 \%$,

- frequency range $f_{c u t}=2[\mathrm{kHz}] \pm 5 \%$,

- resonant frequency $f_{r} \geq 8[\mathrm{kHz}]$.

The accelerometer frequency characteristics were measured for $N=34$ points - Table 1 , while their ordinate values $A(f)$ and $\Phi(f)$ are tabulated in Tables 2 and 3.

Table 1. The measurements points.

$$
\begin{aligned}
f= & {[0.04,0.05,0.06,0.07,0.08,0.09,0.1,0.2,0.3,0.4,0.5,0.6,0.7,0.8,0.9,1.0,1.5,2.0,2.5,3.0,} \\
& 3.5,4.0,4.5,5.0,5.5,6.0,6.5,7.0,7.5,8.0,8.5,9.0,9.5,10.0][\mathrm{kHz}]
\end{aligned}
$$

Table 2. The values of the amplitude characteristic.

$$
\begin{aligned}
A(f)= & {[6.1,6.2,5.7,6.0,6.2,6.3,5.7,5.8,5.7,6.1,5.8,6.2,5.7,6.2,5.7,5.9,6.3,5.9,7.0,6.0,6.9,7.6,} \\
& 8.2,8.8,8.8,10.1,10.2,11.2,13.4,14.2,16.5,14.3,15.5,13.8]\left[\mathrm{mV} / \mathrm{m} \cdot \mathrm{s}^{2}\right]
\end{aligned}
$$

Table 3. The values of the phase characteristic.

$$
\begin{aligned}
\Phi(f)= & {[-0.8,-0.1,-0.1,-0.7,-0.6,-0.4,-0.5,-1.3,-0.2,-0.4,-0.1,-0.1,0.0,0.0,-0.6,-0.2,0.0,} \\
& -0.2,-0.8,-1.6,-1.2,-2.5,-3.3,-6.1,-9.3,-7.3,-10.2,-19.8,-35.6,-25.9,-37.6,-74.4, \\
& -95.6,-97.0] \quad[\mathrm{deg} .]
\end{aligned}
$$

The number of MC trials is assumed to be equal to $10^{5}$, while the means and standard deviations calculated based on the measurement data tabulated in Tables 2-3 are: $E_{A}=8.23$, $E_{\Phi}=-0.22, \sigma_{A}=3.29, \sigma_{\Phi}=0.45$. The uncertainties associated with these measurements are equal to $u\left(A_{\mathrm{n}}\right)=0.57$ and $u\left(\Phi_{n}\right)=0.08$. The estimates of parameters corresponding to the model (38) and the estimates of associated uncertainties are:

and

$$
\widetilde{\boldsymbol{\theta}}=\left[5.95,-1.09 \cdot 10^{-4}, 2.46 \cdot 10^{-8},-1.45 \cdot 10^{-5}, 1.22 \cdot 10^{-8},-4.19 \cdot 10^{-13}\right]
$$

$$
\hat{\boldsymbol{\theta}}=\left[4.03 \cdot 10^{-4}, 6.50 \cdot 10^{-8}, 3.31 \cdot 10^{-10}, 3.92 \cdot 10^{-10}, 5.39 \cdot 10^{-19}, 1.13 \cdot 10^{-25}\right] .
$$

Approximations of the frequency characteristics based both on the measurements tabulated in Tables 2-3 and the results presented by (46) are shown in Fig. 5.

Multiplication by $180 / \pi$ of both the measured data of phase characteristic and the argument calculated for the complex frequency response (38) is necessary to obtain the results expressed in degrees rather than in radians.

Figure 6 shows the error $\Delta$ between the complex frequency responses (3) and (38) which is determined for the measurement data and the estimates of parameters (46) (see Appendix E). 
Based on Fig. 6 it is apparent that he greatest value of fitting error was obtained for the frequency of $8.5 \mathrm{kHz}$ which is close to the resonant frequency.
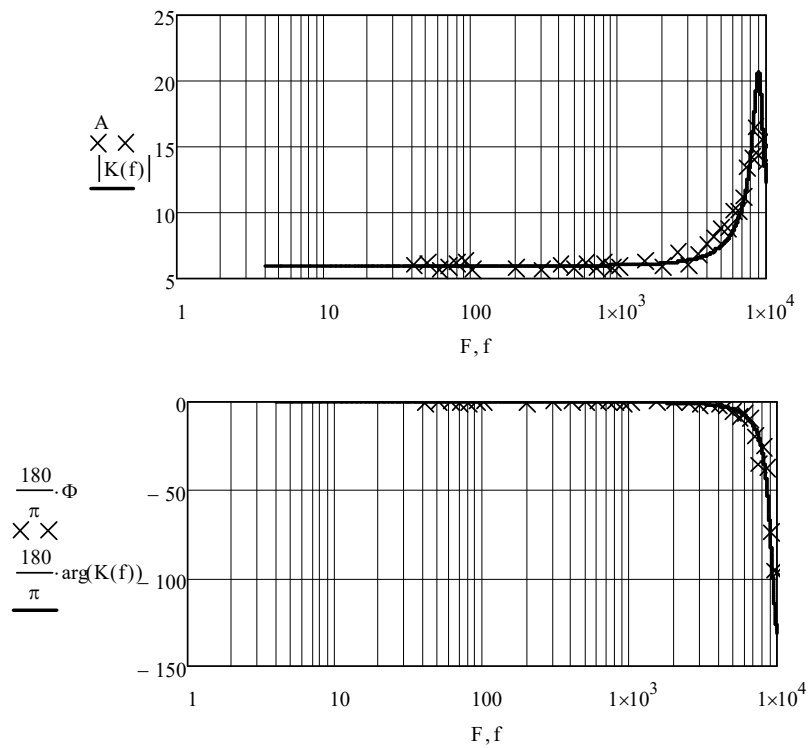

Fig. 5. Approximations of the amplitude and phase characteristics obtained with MathCad.

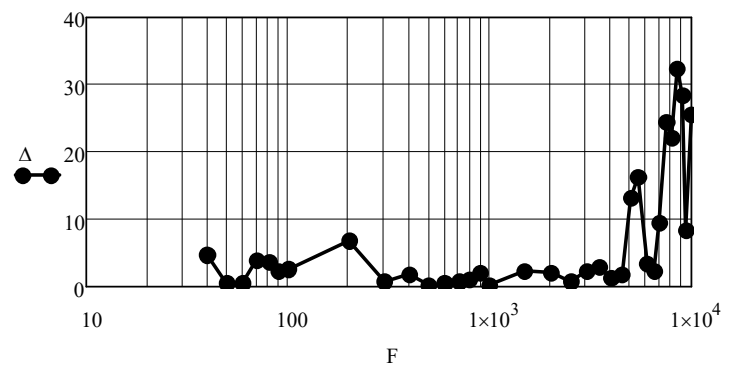

Fig. 6. The fitting error for estimation of accelerometer parameters.

\section{Conclusion}

The main advantage of the solutions presented in this paper is the possibility of modelling a total system related to the charge output accelerometers. The disadvantage is the lack of possibilities to determine the parameters of this accelerometer, but only the functional relations associated with these parameters can be obtained. Furthermore, the numerator of the complex frequency response is extended with a constant value. It follows, that the presented approach to modelling the total system associated with the charge output accelerometer cannot be used for calibration of accelerometers, which requires determination of the voltage sensitivity. 


\section{APPENDIX A}

Calculation of the vector $\mathbf{Y}$ and the matrix $\mathbf{\Psi}$ :

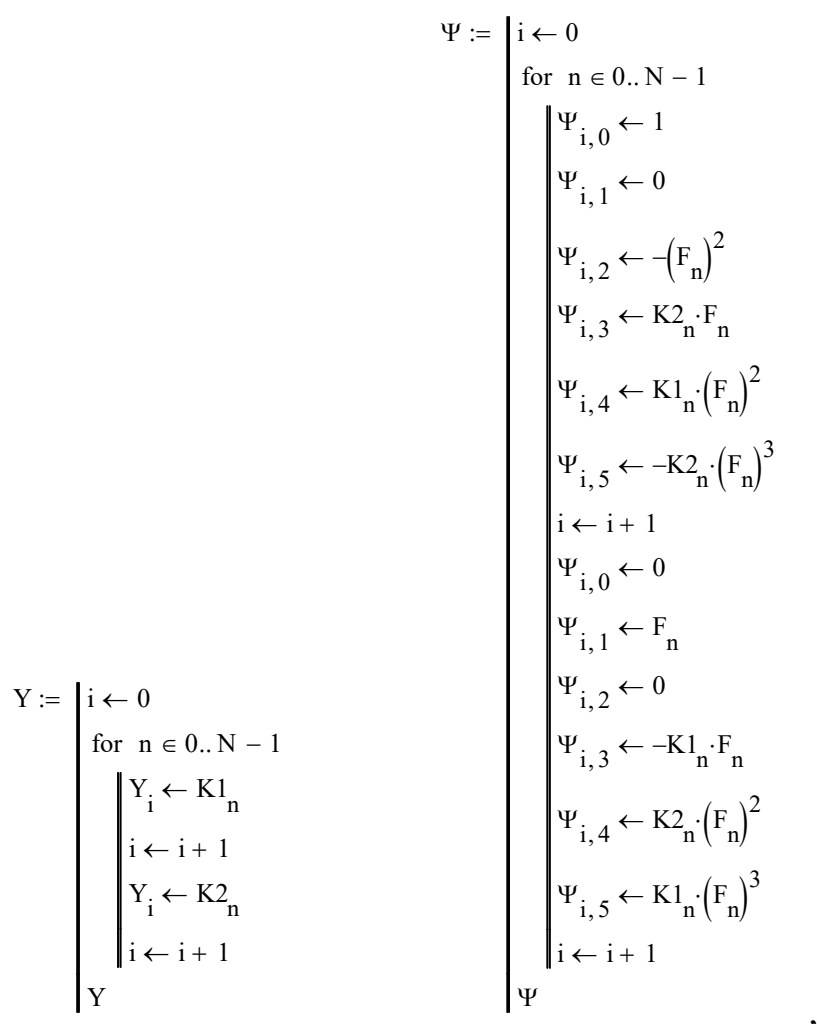

where $\mathrm{K} 1$ and $\mathrm{K} 2$ are the real and imaginary parts of the complex frequency response constructed on the basis of measured amplitude and phase characteristics.

\section{APPENDIX B}

Implementation of the Box-Muller (basic form) algorithm:

$$
\begin{aligned}
& \text { a1 }:=\left(\begin{array}{llll}
11600 & 47003 & 23000 & 33000
\end{array}\right)^{\mathrm{T}} \\
& \mathrm{b} 1:=\left(\begin{array}{llll}
185127 & 45688 & 93368 & 65075
\end{array}\right)^{\mathrm{T}} \\
& \mathrm{c} 1:=\left(\begin{array}{llll}
10379 & 10479 & 19423 & 8123
\end{array}\right)^{\mathrm{T}} \\
& \mathrm{d} 1:=\left(\begin{array}{lllll}
2147483123 & 2147483123 & 2147483123 & 2147483123
\end{array}\right)^{\mathrm{T}}+\left(\begin{array}{llll}
456 & 420 & 300 & 0
\end{array}\right)^{\mathrm{T}}
\end{aligned}
$$




$$
\begin{aligned}
& \varepsilon:=\mid \begin{array}{l}
\text { for } \mathrm{n} \in 0 . . \mathrm{N}-1 \\
\text { for } \mathrm{m} \in 0 . . \mathrm{M}-1
\end{array}
\end{aligned}
$$

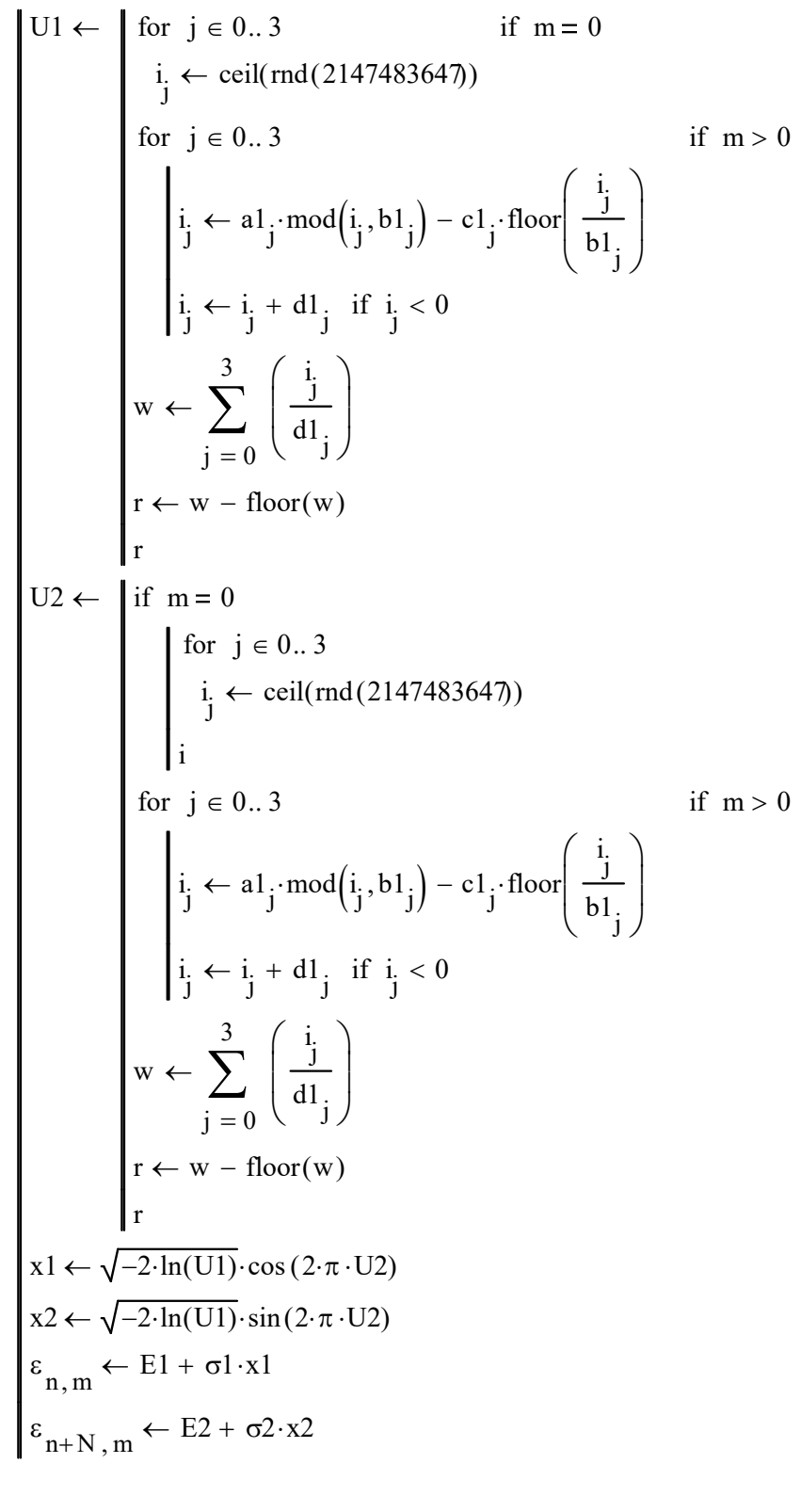

where $E_{1}, \sigma_{1}$ and $E_{2}, \sigma_{2}$ denote the mean and the standard deviation calculated for the vectors $A\left(\omega_{n}\right)$ and $\Phi\left(\omega_{n}\right)$, respectively. 


\section{APPENDIX C}

Calculation of the covariance matrix:

$$
\Sigma:=\mid \begin{aligned}
& \mathrm{V}:=\mathrm{V}^{\mathrm{T}} \\
& \text { for } \mathrm{n} \in 0 . .2 \cdot \mathrm{N}-1 \\
& \mathrm{~s} \leftarrow 0 \\
& \text { for } \mathrm{m} \in 0 . . \mathrm{M}-1 \\
& \mathrm{~s} \leftarrow \mathrm{s}+\mathrm{V}_{\mathrm{m}, \mathrm{n}} \\
& \mathrm{S}_{0, \mathrm{n}} \leftarrow \frac{\mathrm{s}}{\mathrm{M}} \\
& \text { for } \mathrm{n} \in 0 . . \mathrm{N}-1 \\
& \begin{array}{l}
\mathrm{s} \leftarrow 0 \\
\text { for } \mathrm{m} \in 0 . . \mathrm{M}-1 \\
\mathrm{~s} \leftarrow \mathrm{s}+\frac{\left(\mathrm{V}_{\mathrm{m}, \mathrm{n}}-\mathrm{S}_{0, \mathrm{n}}\right)^{2}}{\mathrm{M}^{2}} \\
\Sigma \\
\mathrm{S}_{0, \mathrm{n}} \leftarrow \frac{1}{\mathrm{~s}} \\
\Sigma
\end{array}
\end{aligned}
$$

\section{APPENDIX D}

Calculation of the uncertainties associated with model parameters:

- determination of the covariance matrix

$$
\Sigma_{\mathrm{u}}:=\left(\Psi^{\mathrm{T}} \cdot \Sigma^{-1} \cdot \Psi\right)^{-1}
$$

- determination of the $\mathbf{X}$ matrix associated with the multidimensional normal distribution using the Cholesky decomposition:

$$
X:=\mid \begin{gathered}
\text { for } i \in 0 . . \operatorname{rows}\left(\Sigma_{u}\right)-1 \\
\text { for } j \in 0 . . \operatorname{rows}\left(\Sigma_{u}\right)-1 \\
X_{i, j} \leftarrow 0 \\
\text { for } j \in 0 . . \operatorname{rows}\left(\Sigma_{u}\right)-1 \\
\text { for } i \in j . . \operatorname{rows}\left(\Sigma_{u}\right)-1 \\
\mid \begin{array}{l}
S_{i} \leftarrow \Sigma_{u_{i, j}} \\
\text { for } k \in 0 . . j \\
S_{i} \leftarrow S_{i}-X_{j, k} \cdot X_{i, k} \\
X_{i, j} \leftarrow \frac{S_{i}}{\sqrt{S_{j}}}
\end{array}
\end{gathered}
$$

- determination of the uncertainty vector: 
$\Delta \varepsilon:=\mid \begin{gathered}\text { for } \mathrm{m} \in 0 . . \mathrm{M}-1 \\ { }_{\Delta \varepsilon}^{\langle\mathrm{m}\rangle} \leftarrow \mathrm{X}^{\mathrm{T}} \cdot \varepsilon \mathrm{m}^{\langle\mathrm{m}\rangle} \\ \Delta \varepsilon\end{gathered}$

where $\varepsilon^{<\mathrm{m}>}$ is a six-element random vector determined using the Box-Muller algorithm (Appendix A).

- determination of the vector (43):

$\Theta 1:=$ for $\mathrm{m} \in 0 . . \mathrm{M}-1$

$$
\left.\right|_{\Theta 1} ^{\Theta 1}\left\langle{ }^{\langle\mathrm{m}\rangle} \leftarrow \Theta+\Delta \varepsilon{ }^{\langle\mathrm{m}\rangle}\right.
$$

- calculation of the covariance matrix (45) and uncertainties $\hat{\boldsymbol{\theta}}$ associated with the parameters (39):

$$
\begin{aligned}
& \Theta 1:=\Theta 1{ }^{\mathrm{T}} \\
& \Sigma_{\Theta}:=\mid \begin{array}{l}
\text { for } \mathrm{m} \in 0 . . \mathrm{M}-1 \\
\| \begin{array}{l}
\mathrm{s} \leftarrow 0 \\
\text { for } \mathrm{n} \in 0 . . \mathrm{N} 1-1 \\
\mathrm{~s} \leftarrow \mathrm{s}+\Theta 1_{\mathrm{n}, \mathrm{m}} \\
\mathrm{S}_{0, \mathrm{~m}} \leftarrow \frac{\mathrm{s}}{\mathrm{N} 1}
\end{array} \\
\text { for } \mathrm{m} \in 0 . . \mathrm{M}-1 \\
\text { for } \mathrm{p} \in 0 . . \mathrm{M}-1 \\
\begin{array}{l}
\mathrm{s} \leftarrow 0 \\
\text { for } \mathrm{n} \in 0 . . \mathrm{N} 1-1
\end{array} \\
\mathrm{~s} \leftarrow \mathrm{s}+\frac{\left(\Theta 1 \mathrm{n}, \mathrm{m}-\mathrm{S}_{0, \mathrm{~m}}\right)^{2}}{\mathrm{~N} 1} \\
\Theta 2 \mathrm{p}, \mathrm{m} \leftarrow \sqrt{\mathrm{s}} \\
\Theta 2 \leftarrow \operatorname{diag}(\Theta 2)
\end{array}
\end{aligned}
$$

\section{APPENDIX E}

Determination of the difference between the complex frequency responses (3) and (38):

$$
\Delta:=\mid \begin{aligned}
& \text { for } \mathrm{n} \in 0 . . \mathrm{N}-1 \\
& \mid \begin{array}{l}
\mathrm{A} 1_{\mathrm{n}} \leftarrow\left|\mathrm{K}\left(\mathrm{F}_{\mathrm{n}}, \mathrm{a} 0, \mathrm{a} 1, \mathrm{a} 2, \mathrm{~b} 1, \mathrm{~b} 2, \mathrm{~b} 3\right)\right| \\
\Phi 1_{\mathrm{n}} \leftarrow \frac{180}{\pi} \cdot \arg \left(\mathrm{K}\left(\mathrm{F}_{\mathrm{n}}, \mathrm{a} 0, \mathrm{a} 1, \mathrm{a} 2, \mathrm{~b} 1, \mathrm{~b} 2, \mathrm{~b} 3\right)\right) \\
\left.\mathrm{K} 1_{\mathrm{n}} \leftarrow \mathrm{A} 1_{\mathrm{n}} \cdot \mathrm{e}^{(\mathrm{i} \cdot \Phi} \mathrm{1}_{\mathrm{n}}\right) \\
\left.\left.\mathrm{K} 2_{\mathrm{n}} \leftarrow \mathrm{A}_{\mathrm{n}} \cdot \mathrm{e}^{\mathrm{i} \cdot\left(\frac{180}{\pi} \cdot \Phi\right)}\right)_{\mathrm{n}}\right] \\
\Delta_{\mathrm{n}} \leftarrow\left|\mathrm{K} 1_{\mathrm{n}}-\mathrm{K} 2_{\mathrm{n}}\right|
\end{array}
\end{aligned}
$$




\section{References}

[1] Yu, J.Ch., Lan, Ch.B. (1999). System Modeling and Robust Design of Microaccelerometer using Piezoelectric Thin Film. Proc. of the IEEE Int. Conf. on MFI for Intell. Syst., 99-104.

[2] Levy, E.C. (1959). Complex-Curve Fitting. IEEE T. Automat. Contr., AC-4, 37-44.

[3] Sanathanan, C.K., Koerner, J. (1963). Transfer Function Synthesis as a Ratio of Two Complex Polynomials. IEEE T. Automat. Contr., AC-9, 56-58.

[4] Strobel, H. (1966). On a New Method of Determining the Transfer Function by Simultaneous Evaluation of the Real and Imaginary Parts of the Measured Frequency Response, 3-rd IFAC Symposium. London.

[5] Gyürki, J. (1970). Some Questions of Identification on the basis of Frequency Response. Acta Tech. Hung., $68,145-160$.

[6] Payne, P.A. (1970). An Improved Technique for Transfer Function Synthesis from Frequence Response Data. IEEE T. Automat. Contr., 14, 480-483.

[7] t'Mannetje, J.J. (1973). Transfer-Function Identification using a Complex Curve-Fitting Technique. J. Mech. Eng. Sci., 15, 339-345.

[8] Stahl, H. (1984). Transfer Function Synthesis using Frequency Response Data. Int. J. Control, 39, 541-550.

[9] Whitfield, A.H. (1987). Asymptotic Behavior of Transfer Function Synthesis Methods. Int. J. Control, 10831092.

[10] Glob, G.H., Van Loan, C.F. (1980). An Analysis of the Total Least Squares Problem. SIAM J. Numer. Anal., $17,883-893$.

[11] Pintelon, R., Schoukens, J. (2001). System Identification: A Frequency Domain Approach. IEEE Press, Piscataway, New York.

[12] JCGM 101 (2008). Evaluation of Measurement Data - Supplement 1 to the Guide to the Expression of Uncertainty in Measurement - Propagation of Distributions using a Monte Carlo Method.

[13] Soysal, A.O., Semlyen, A. (1993). Practical Transfer Function Estimation and its Application to Wide Frequency Range Representation of Transformers. IEEE T. Power Deliver., 8, 1627-1637.

[14] Janiszowski, K.B. (2014). Approximation of a Linear Dynamic Process Model using the Frequency Approach and a Nonquadratic Measure of the Model Error. Int. J. Appl. Math. Comput. Sci., 24, 99-109.

[15] Link, A., Täbner, A., Wabinski, W., Bruns, T., Elster, C. (2007). Modelling Accelerometers for Transient Signals using Calibration Measurement upon Sinusoidal Excitation. Measurement, 40, 928-935.

[16] Tomczyk, K., Sieja, M. (2006). Acceleration transducers calibration based on maximum dynamic error. Technical Trans., 3E, 37-49.

[17] Layer, E., Tomczyk, K. (2010). Measurements. Modelling and Simulation of Dynamic Systems. SpringerVerlag, Berlin Heidelberg.

[18] Tomczyk, K., Layer, E. (2015). Accelerometer Errors in Measurements of Dynamic Signals. Measurement, 60, 292-298.

[19] JCGM 100 (2008). Evaluation of measurement data - Guide to the Expression of Uncertainty in Measurement.

[20] Magain, P., Courbin, F., Sohy, S. (1988). Deconvolution with Correct Sampling. The Astrophys. J., 494, 472-477.

[21] Morawski, R., Szczecinski, L., Barwicz, A. (1995). Deconvolution algorithms for instrumental applications: A comparative study. Journal of Chemometr., 9(1), 3-20. 